\title{
Magnetoelastic phase diagram of TbNi2B2C
}

Toft-Petersen, Rasmus; Jensen, Thomas Bagger Stibius; Jensen, Jens; von Zimmermann, Martin; Sloth, Steffen; Isaksen, Frederik Werner; Christensen, Niels Bech; Chen, Yunzhong; Siemensmeyer, Konrad; Kawano-Furukawa, Hazuki

Total number of authors:

13

Published in:

Physical Review B

Link to article, DOI:

10.1103/PhysRevB.97.224417

Publication date:

2018

Document Version

Publisher's PDF, also known as Version of record

Link back to DTU Orbit

Citation (APA):

Toft-Petersen, R., Jensen, T. B. S., Jensen, J., von Zimmermann, M., Sloth, S., Isaksen, F. W., Christensen, N. B., Chen, Y., Siemensmeyer, K., Kawano-Furukawa, H., Takeya, H., Abrahamsen, A. B., \& Andersen, N. H. (2018). Magnetoelastic phase diagram of $\mathrm{TbNi}_{2} \mathrm{~B}_{2} \mathrm{C}$. Physical Review B, 97(22), [224417]. https://doi.org/10.1103/PhysRevB.97.224417

\section{General rights}

Copyright and moral rights for the publications made accessible in the public portal are retained by the authors and/or other copyright owners and it is a condition of accessing publications that users recognise and abide by the legal requirements associated with these rights.

- Users may download and print one copy of any publication from the public portal for the purpose of private study or research.

- You may not further distribute the material or use it for any profit-making activity or commercial gain

- You may freely distribute the URL identifying the publication in the public portal 


\title{
Magnetoelastic phase diagram of $\mathrm{TbNi}_{2} \mathrm{~B}_{2} \mathrm{C}$
}

Rasmus Toft-Petersen, ${ }^{1}$ Thomas Bagger Stibius Jensen, ${ }^{1}$ Jens Jensen, ${ }^{2}$ Martin von Zimmermann, ${ }^{3}$ Steffen Sloth, ${ }^{1}$ Frederik Werner Isaksen, ${ }^{1}$ Niels Bech Christensen, ${ }^{1}$ Yunzhong Chen, ${ }^{4}$ Konrad Siemensmeyer, ${ }^{5}$ Hazuki Kawano-Furukawa,,${ }^{6,7}$ Hiroyuki Takeya, ${ }^{8}$ Asger Bech Abrahamsen, ${ }^{9}$ and Niels Hessel Andersen ${ }^{1}$

${ }^{1}$ Department of Physics, Technical University of Denmark, DK-2880 Kgs. Lyngby, Denmark

${ }^{2}$ Niels Bohr Institute, Universitetsparken 5, DK-2100 Copenhagen, Denmark

${ }^{3}$ Deutsches Elektronen-Synchrotron DESY, D-22603 Hamburg, Germany

${ }^{4}$ Department of Energy Conversion and Storage, Technical University of Denmark, DK-4000 Roskilde, Denmark

${ }^{5}$ Helmholtz Zentrum Berlin für Materialien und Energie, D-14109 Berlin, Germany

${ }^{6}$ Division of Natural/Applied Science, Graduate School of Humanities and Science, Ochanomizu University, Bunkyo-ku, Tokyo 112-8610, Japan

${ }^{7}$ RIKEN Center for Emergent Matter Science (CEMS), 2-1 Hirosawa, Wako, Saitama, 351-0198, Japan

${ }^{8}$ National Institute for Materials Science, 1-2-1 Sengen, Tsukuba, Ibaraki, 305-0047, Japan

${ }^{9}$ Department of Wind Energy, Technical University of Denmark, DK-4000 Roskilde, Denmark

(Received 12 April 2018; revised manuscript received 25 May 2018; published 15 June 2018)

\begin{abstract}
The magnetic phase diagram of the quaternary borocarbide $\mathrm{TbNi}_{2} \mathrm{~B}_{2} \mathrm{C}$ is investigated by direct means and by studying magnetically induced modifications of the crystal structure. Detailed superconducting quantum interference device measurements reveal a complex phase diagram with five distinct magnetic phases. The phase boundaries are mapped out comprehensively. Synchrotron hard x-ray measurements in applied magnetic fields are employed to probe the magnetoelastic distortions throughout the phase diagram. The determination of the wave vectors of these field-induced lattice deformations suggests a range of commensurate spin-slip-type magnetic structures at low temperatures with wave vectors of the form $(q, 0,0)$ with $q=6 / 11$ and 5/9. The proposed magnetic structures yield values of magnetization well in-line with observations. The scattering intensity due to the magnetoelastic deformations exhibits a drastic jump at the phase boundary at $1.3 \mathrm{~T}$ and low temperatures.
\end{abstract}

DOI: 10.1103/PhysRevB.97.224417

\section{INTRODUCTION}

Since its discovery $[1-4]$ the family of quaternary nickel borocarbides $R \mathrm{Ni}_{2} \mathrm{~B}_{2} \mathrm{C}(R=\mathrm{Y}$ and the rare earths) have attracted attention for a variety of reasons. Displaying a great abundance of superconducting and magnetic phases with similar energy scales for the two phenomena, the nickel borocarbides have been a diverse testing ground for studying the interplay between the two $[5,6]$. Specifically, the coexistence of superconductivity and magnetism is found in the systems where $R$ is Dy, Ho, Er, or Tm [1,3], where the superconducting transition temperature scales inversely with the de Gennes factor, $d G=\left(g_{J}-1\right)^{2} J(J+1)$, indicative of competing magnetic and superconducting order parameters [5]. $\mathrm{ErNi}_{2} \mathrm{~B}_{2} \mathrm{C}$ is of special interest, exhibiting a superconducting transition at $T_{c}=11 \mathrm{~K}$ [5], antiferromagnetic (AFM) ordering at $T_{N}=6 \mathrm{~K}$, and weak ferromagnetism (WFM) below $T_{\mathrm{WFM}}=2.3 \mathrm{~K}$ [7-10]. $\mathrm{ErNi}_{2} \mathrm{~B}_{2} \mathrm{C}$ is thus one of the rare instances of microscopic coexistence of ferromagnetism and superconductivity $[11,12]$, a recurring topic in condensed matter physics $[13,14]$-also found in the case of $\mathrm{UGe}_{2}[15]$ and in crystalline superconducting nanowires [16].

A further testament to the complexity of the nickel borocarbides is the presence of a magnetoelastic coupling, where the AFM order parameter gives rise to lattice distortions [17-19]. Several of the borocarbides systems exhibit spin density wave (SDW) magnetic order with propagation vector $\boldsymbol{q} \sim(0.55,0,0)[20,21]$. The recurrence of this ordering vector is likely due to the indirect Ruderman-Kittel-KasuyaYoshida (RKKY) coupling in cooperation with a Fermi surface nesting producing a maximum of the generalized electronic susceptibility at $\boldsymbol{q} \sim(0.6,0,0)[22,23]$. In $\mathrm{TmNi}_{2} \mathrm{~B}_{2} \mathrm{C}$, for instance, field-dependent lattice distortions were discovered using hard x-ray diffraction in longitudinal fields [24]. The distortions are prominent above $2 \mathrm{~T}$ in a phase with magnetic ordering vector $(0.484,0,0)$, in contrast to the weaker coupling observed in the low field magnetic phase with ordering vector $(0.094,0.094,0)$ [25]. In Ref. [24], the field dependency of the lattice distortions was shown to be the result of a quadrupolar phase, where magnetoelasticity is enchanced by a soft phonon mode $\Delta_{4}$, important for the electron-phonon coupling and hence superconductivity in the nickel borocarbides [26-28].

$\mathrm{TbNi}_{2} \mathrm{~B}_{2} \mathrm{C}$ is nonsuperconducting, at least, above $300 \mathrm{mK}$ $[29,30]$. The crystal structure is tetragonal at room temperatures with lattice parameters $a=b=3.5435 \AA$ and $c=$ $10.4295 \AA$ [31]. The $\mathrm{Tb}^{3+}$ ion has a ${ }^{7} \mathrm{~F}_{6}$ ground state with $S=3, L=3, J=6$, and $g_{J}=3 / 2$. Below $T_{N}=15 \mathrm{~K}$ a spin density wave (SDW) sets in, polarized along $\boldsymbol{a}$ parallel to the magnetic modulation vector $\boldsymbol{q}=(q, 0,0)[20,21,32-34]$. The magnetic modulation varies from 0.552 at $15 \mathrm{~K}$ to $q \simeq 0.545$ at $2.3 \mathrm{~K}$, where the SDW is significantly squared up [20]. $\mathrm{TbNi}_{2} \mathrm{~B}_{2} \mathrm{C}$ develops weak ferromagnetism below $T_{\mathrm{WFM}}=8 \mathrm{~K}$ [20,29,35-37]. At the Néel temperature, the system undergoes a structural phase transition from tetragonal to orthorhombic crystal structure [38]. For fields along [100], the phase diagram 
of $\mathrm{TbNi}_{2} \mathrm{~B}_{2} \mathrm{C}$ is exceedingly complex [19]. The crystal studied in Ref. [37] was found to be divided in two magnetic domains in the weak ferromagnetic phase below $8 \mathrm{~K}$ at zero applied field. The ordering wave vector $\boldsymbol{q}=(0.545,0,0)$ is constant in the dominating domain, whereas in the smaller one, $\boldsymbol{q}$ depends on temperature being about $(0.548,0,0)$ at $T_{\mathrm{WFM}}$ and equal to $(0.550, \pm 0.002,0)$ in the zero temperature limit.

The low energy $\Delta_{4}$ mode was detected in $\mathrm{TbNi}_{2} \mathrm{~B}_{2} \mathrm{C}[39,40]$, and shown to exhibit significant softening when cooling from 300 to $30 \mathrm{~K}$ [40]. This testifies to an electron-phonon coupling in $\mathrm{TbNi}_{2} \mathrm{~B}_{2} \mathrm{C}$ of similar magnitude as in the superconducting counterparts. In Ref. [40], it was therefore suggested that the lack of superconductivity in $\mathrm{TbNi}_{2} \mathrm{~B}_{2} \mathrm{C}$ is due to pair breaking caused by the strong magnetic coupling, rather than a result of a weak electron-phonon coupling.

Here we investigate the possible impact of the $\Delta_{4}$ mode on the lattice distortions, by probing the magnetoelastic phase diagram of $\mathrm{TbNi}_{2} \mathrm{~B}_{2} \mathrm{C}$. Using VSM and superconducting quantum interference device (SQUID) magnetometry, we study the complex magnetic phase diagram of $\mathrm{TbNi}_{2} \mathrm{~B}_{2} \mathrm{C}$ for fields along [100]. We proceed to probe the magnetoelastic distortions, and find a weak modulated lattice distortion at $2 \boldsymbol{q}=(1.091,0,0)$ below $1 \mathrm{~T}$ at the lowest temperatures. Upon crossing a phase boundary at $1.3 \mathrm{~T}$, this magnetoelastic response increases drastically. Via a first-order phase transition at about $2.1 \mathrm{~T}$, $\mathrm{TbNi}_{2} \mathrm{~B}_{2} \mathrm{C}$ enters yet another magnetic phase, where strong distortions at a slightly different wave vector $2 \boldsymbol{q}=(1.110,0,0)$ is detected. The lattice displacements are not along the $c$ axis as found in the quadrupolar phase of superconducting $\mathrm{TmNi}_{2} \mathrm{~B}_{2} \mathrm{C}$, but along the $a$ axis. So while $\Delta_{4}$ is crucial for understanding the magnetoelastic deformations in $\mathrm{TmNi}_{2} \mathrm{~B}_{2} \mathrm{C}$, the magnetoelastic Hamiltonian in $\mathrm{TbNi}_{2} \mathrm{~B}_{2} \mathrm{C}$ looks to be more similar to that of $\mathrm{ErNi}_{2} \mathrm{~B}_{2} \mathrm{C}[19,41]$.

\section{EXPERIMENTAL DETAILS}

For magnetization measurement, initial investigations were performed on a CRYOGENIC cryogen free measurement system (CFMS) by performing vibrating sample magnetization (VSM) measurements. A high-quality single crystal measuring $1 \times 1 \times 3 \mathrm{~mm}^{3}$ was used to perform magnetization measurements in the temperature interval $2-30 \mathrm{~K}$ and field interval 0-5 T along the $a$ axis. After identifying the regions of interest and recognizing the complexity of the phase diagram, we turned to using a superconducting quantum interference device (SQUID). The crystal size was $1 \times 1 \times 2 \mathrm{~mm}^{3}$. In the temperature scans, all transitions were continuous, and we used the second derivative $\frac{d^{2} M}{d T^{2}}$ to estimate phase boundaries. In the field scans, the phase transitions were estimated as being of first order, and accordingly we used peaks in the first derivative $\frac{d M}{d H}$ to estimate the phase boundaries. In the case of the field scans, the only exception is the phase boundary to the saturated paramagnetic phase, where we used the saturation point as a criterion. This is due to the fact that the saturated phase is defined as containing no AFM component. An example of a field scan is shown in Fig. 1 while the phase diagram measured by magnetization is shown in Fig. 2 .

The magnetoelastic response in the form of structural distortions was examined in $\mathrm{TbNi}_{2} \mathrm{~B}_{2} \mathrm{C}$ as a function of temperature and magnetic field along [100] in the whole phase diagram,

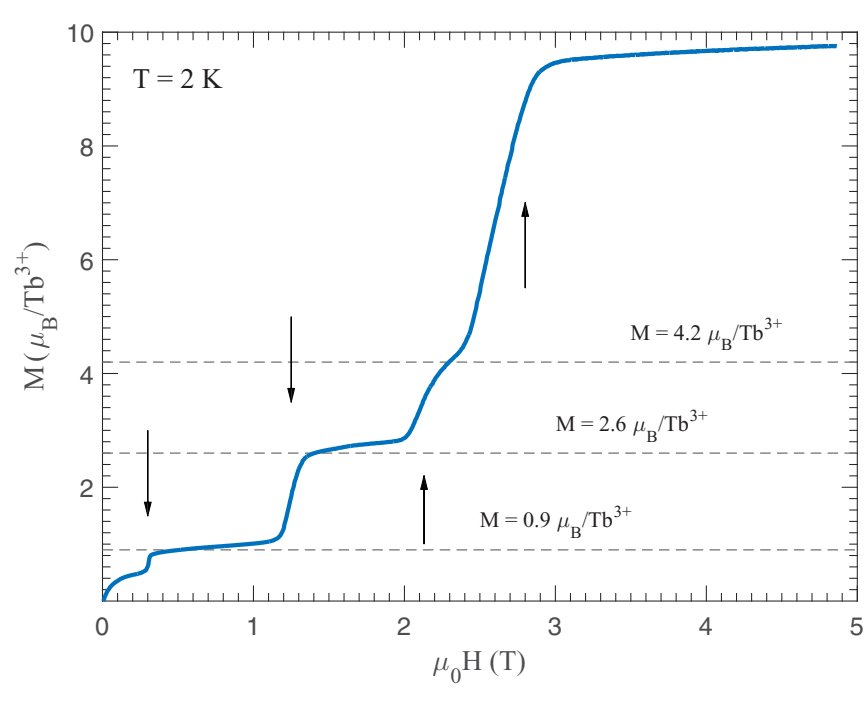

FIG. 1. Magnetization of $\mathrm{TbNi}_{2} \mathrm{~B}_{2} \mathrm{C}$ as function of magnetic field applied along the $a$ axis after cooling to $2 \mathrm{~K}$. There are four magnetic phase transitions before gradual saturation occurs above $2.8 \mathrm{~T}$. The errorbars are smaller than the width of the curve.

using elastic synchrotron x-ray diffraction. The experiments were performed on the triple-axis diffractometer beamline BW5 at DESY in Hamburg. The sample was a single crystal $\mathrm{TbNi}_{2} \mathrm{~B}_{2} \mathrm{C}$ of approximate dimensions $1 \times 1 \times 3.5 \mathrm{~mm}^{3}$ (weight $\sim 25 \mathrm{mg}$ ) and placed in a 10 tesla horizontal cryomagnet with the $(a b)$ plane as scattering plane. The analyzer and monochromator were (111) $\mathrm{Si} / \mathrm{Ge}$-gradient crystals set to reflect photons of energy $99.818 \mathrm{keV}(\lambda=0.1242 \AA)$.

The measurements consisted of several field and temperature scans. For each temperature and field we would first align by centering on the $(2,0,0)$ Bragg peak using $\omega$ and $\theta-2 \theta$ scans

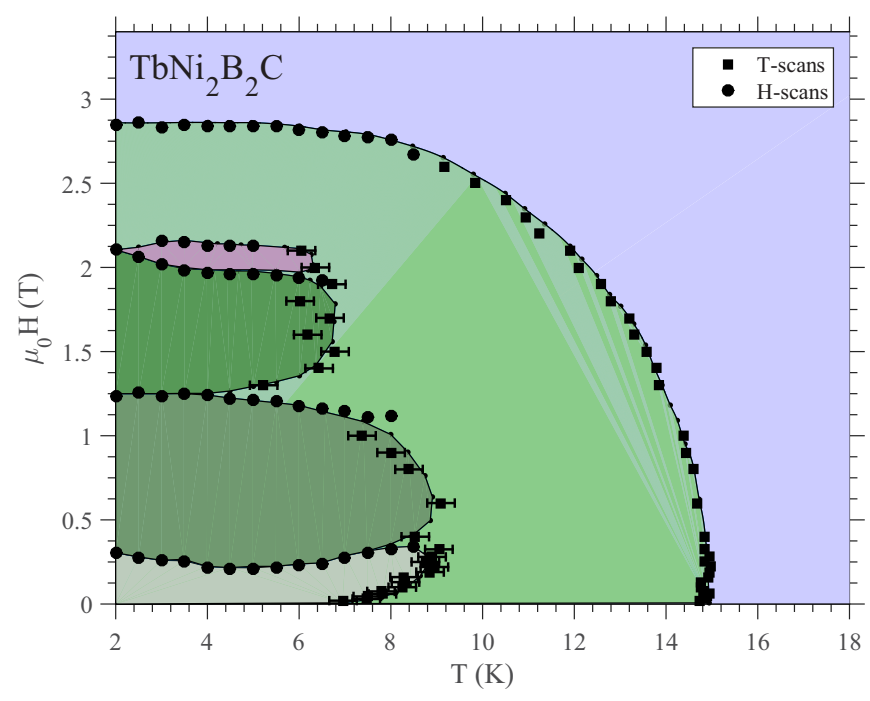

FIG. 2. Magnetic phase diagram of $\mathrm{TbNi}_{2} \mathrm{~B}_{2} \mathrm{C}$ as determined from magnetization measurements, for magnetic fields along the [100] axis. Five magnetic phases are evident below the saturation field of $\mu_{0} H=2.8 \mathrm{~T}$. All temperature scans are field cooling and all field scans are for increasing field from $0-5 \mathrm{~T}$ (zero field cooling). Lines are guides to the eye based on the analysis of the raw data. 


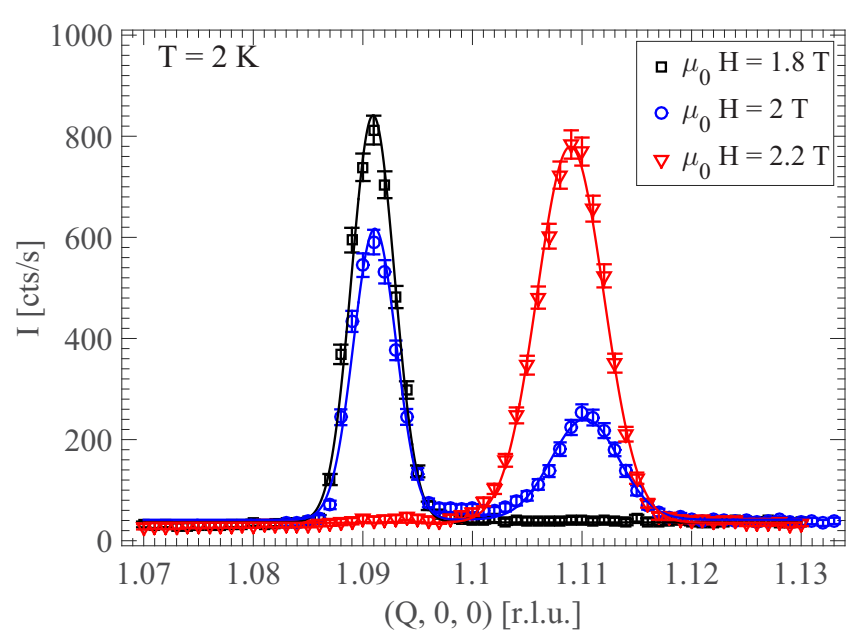

FIG. 3. X-ray scattering intensity along a scan in reciprocal space $(Q, 0,0)$ at $T=2 \mathrm{~K}$ for $\boldsymbol{H} \| \boldsymbol{a}$. For fields below $2 \mathrm{~T}$, a single peak appears at $Q 1=(1.091,0,0)$. Above $2 \mathrm{~T}$ the peak position has shifted to $Q 2=(1.110,0,0)$, though at $2 \mathrm{~T}$ both peaks are present.

to ensure the correct relative position between instrument and sample. Then followed a longitudinal scan along $(Q, 0,0)$ to identify the satellite peaks arising from magnetoelastic distortions. Upon discovering a peak, we performed a transverse scan around the peak position to record the intensity. The sample was zero-field cooled down to the desired temperature from above $T_{N}$ (typically from $20 \mathrm{~K}$ ). Keeping the temperature constant we would then ramp the field to its target, and at the target field perform the scanning procedure described above. This was done for several fields up to 3 tesla, where the satellite peaks were no longer observed. All temperature scans were performed under field cooling conditions. For every measurement we would extract the positions and integrated intensities of the peaks by fitting the $(Q, 0,0)$-scan with Gaussian peak shapes, as illustrated in Fig. 3.

\section{MAGNETIC PHASE DIAGRAM}

To probe the complex phase diagram of $\mathrm{TbNi}_{2} \mathrm{~B}_{2} \mathrm{C}$, a dense set of field and temperature scans were performed on the SQUID device. The measured Néel temperature of $T_{N} \simeq$ $14.8 \mathrm{~K}$ and the weak ferromagnetic transition at $T_{\mathrm{WFM}} \simeq 7.5 \mathrm{~K}$ is in good agreement with those reported in Ref. [37]. Field scans from 0-5 $\mathrm{T}$ (zero field cooled) were performed in the temperature interval 2-15 K (see Fig. 1). All field scans revealed a very complex phase diagram below $T_{\mathrm{WFM}} \simeq 8 \mathrm{~K}$ and a simple one for $T>T_{\mathrm{WFM}}$. At $2 \mathrm{~K}$, four magnetic transitions are evident, while for all field scans at temperatures above $T=9 \mathrm{~K}$, only the transition to the high-field paramagnetic phase occurs. In the temperature interval 2-9 K, between 3 and 5 phase transitions are evident in the field scans.

The phase diagram of $\mathrm{TbNi}_{2} \mathrm{~B}_{2} \mathrm{C}$ as determined from the SQUID measurements is shown in Fig. 2. The phase boundaries presented here are consistent with those presented in Ref. [19] where the low field boundary below $0.5 \mathrm{~T}$ is added by our studies. The complexity of the phase diagram of $\mathrm{TbNi}_{2} \mathrm{~B}_{2} \mathrm{C}$ is limited to temperatures below $9 \mathrm{~K}$, above which the fieldand temperature dependencies are similar to those of a simple antiferromagnet. In Ref. [19], the phase transition at 2.1 T was conjectured to be due to a change in the modulation of the magnetic structure. This proposition is supported by our x-ray measurements presented in the next section.

\section{X-RAY EXPERIMENTS}

To investigate the magnetoelastic response of $\mathrm{TbNi}_{2} \mathrm{~B}_{2} \mathrm{C}$, we performed $\mathrm{x}$-ray diffraction experiments focusing on wave vectors related to the magnetic ordering wave vector $\boldsymbol{q}=$ $(0.545,0,0)$ determined at $2 \mathrm{~K}$ in Ref. [37]. Following the approach in Ref. [24], we searched for peaks at positions $(n q, 0, L)$, where $n$ and $L$ are integers. In contrast to $\mathrm{TmNi}_{2} \mathrm{~B}_{2} \mathrm{C}$, no intensity was found at $(q, 0,8)$. For $L=0$ and $n=2$, however, we found a peak of strongest intensity at $2 \boldsymbol{q}=$ $(1.091,0,0) \equiv Q 1$ below $2 \mathrm{~T}$. Above $2 \mathrm{~T}$, the position of the satellite peak position shifted to $2 \boldsymbol{q}=(1.110,0,0) \equiv Q 2$ at 2 $\mathrm{K}$, indicative of magnetic ordering vector of $\boldsymbol{q}=(0.555,0,0)$ above $2 \mathrm{~T}$. The uncertainty in the determination of the peak position along $\boldsymbol{a}^{*}$ is around 0.002 . Since the resolution of the hard x-ray diffractometer is relaxed in the plane perpendicular to the scattering vector, any peaks with a small $\boldsymbol{b}^{*}$ component of the order of \pm 0.002 are picked up in the scans along $(Q, 0,0)$. Since it is unresolved by our experiment, we omit this component in our description of the wave vectors. As shown in Fig. 3, the two wave vectors, $Q 1$ and $Q 2$, coexist in a narrow interval around the first-order transition at $2 \mathrm{~T}$. Since the $\mathrm{x}$-ray diffraction cross section only probes lattice distortions along the scattering vector $(Q, 0,0)$, the modulated distortions observed are along $\boldsymbol{a}$ in $\mathrm{TbNi}_{2} \mathrm{~B}_{2} \mathrm{C}$ and not along $c$ as in $\mathrm{TmNi}_{2} \mathrm{~B}_{2} \mathrm{C}$. The low-field data showed no sign of the smaller domain at $\boldsymbol{q}=(0.550, \pm 0.002,0)$ observed at zero field in Ref. [37] at $2 \mathrm{~K}$. However, the observed peak position at $Q 1=(1.091,0,0)$ in low fields predicts a magnetic ordering wave vector, which is in perfect agreement with the commensurable value $q=\frac{6}{11}=0.54545$ derived in Ref. [37] for the main magnetic domain at zero field.

At zero field we observed no peak intensity below $5 \mathrm{~K}$, while above $5 \mathrm{~K}$ a weak intensity peak was found at $(Q, 0,0)$, with $Q$ between 1.09 and 1.11 , moderately growing with temperature until $T_{N}$, where the intensity dropped to zero. At $T=2 \mathrm{~K}$, a magnetoelastic distortion appears at $\mu_{0} H=0.25 \mathrm{~T}$, leading to a weak reflection at the $Q 1$ position. The intensity grows slightly with field until the phase transition at $\mu_{0} H=1.3 \mathrm{~T}$, where the intensity of the X-ray peak drastically increases (see Fig. 4). The intensity remains roughly constant with field up to the phase boundary at $2 \mathrm{~T}$, where the structural ordering wave vector changes from $Q 1$ to $Q 2$, indicative of a shift in the magnetic ordering vector from $\boldsymbol{q} \sim\left(\frac{6}{11}, 0,0\right)$ to $\boldsymbol{q} \sim\left(\frac{5}{9}, 0,0\right)$. The abruptness of the change and the constancy of the wave vectors on both side of the transition strongly indicated that this is a first-order transition between two commensurably ordered magnetic phases. The maximum in the scattering intensity, corresponding to 2.6 in the intensity units applied in Figs. 4 and 5 , is estimated to correspond to a relative displacement of the Tb ions by $E_{11}(2 q)=7 \cdot 10^{-4}$, where the modulated strain of the $\mathrm{Tb}$ ion at site $\boldsymbol{R}_{i}$ is defined by

$$
E_{11}\left(\boldsymbol{R}_{i}\right)=\frac{u_{1}\left(\boldsymbol{R}_{i}\right)}{a / 2}=E_{11}(2 \boldsymbol{q}) \cos \left(2 \boldsymbol{q} \cdot \boldsymbol{R}_{i}+\phi\right)
$$




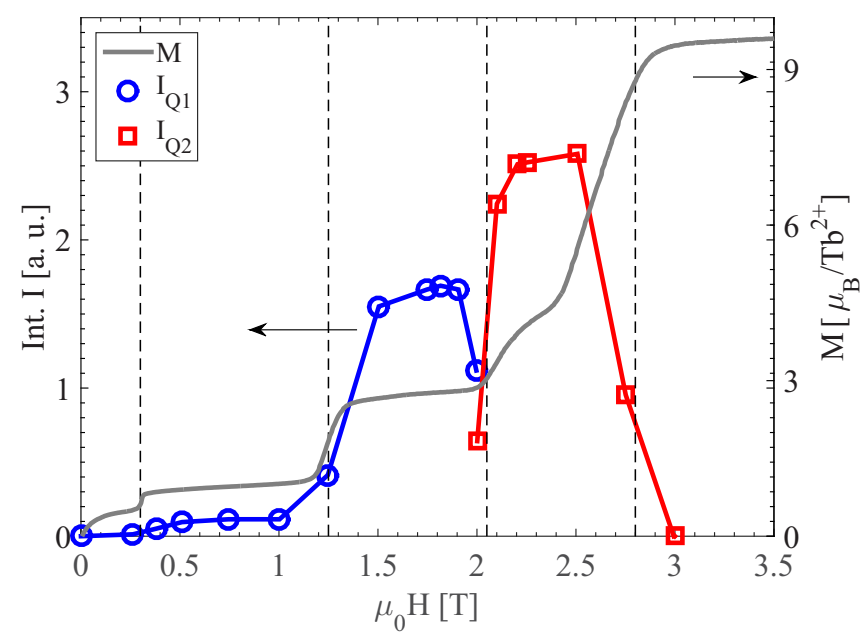

FIG. 4. Intensity $I$ of the x-ray peaks (at $T=2 \mathrm{~K}$ ), signifying the magnitude of the magnetoelastic lattice distortion at $2 \boldsymbol{q}$ plotted alongside the magnetization $M$ (at $T=1.7 \mathrm{~K}$ ) as measured by the VSM system, as a function of applied magnetic field $\boldsymbol{H} \| \boldsymbol{a}$. A drastic increase in intensity is evident at the magnetic phase transition at $1.3 \mathrm{~T}$, whereas the ordering wave vector changes abruptly at the transition at $2 \mathrm{~T}$ (see Fig. 3). The errorbars on both datasets are smaller than the markers.

in terms of the displacement vector component along the 1 axis parallel to $\boldsymbol{a}$ divided by the distance $a / 2$ between the $(b c)$ layers of $\mathrm{Tb}$ ions.

The similar first-order magnetoelastic transition, at about $2 \mathrm{~T}$ at $2 \mathrm{~K}$, is also observed at 4 and $6 \mathrm{~K}$, albeit the possible commensurability of the underlying magnetic order above $2 \mathrm{~T}$ becomes less obvious the higher the temperature is. The results obtained at $6 \mathrm{~K}$ are shown in Fig. 5. At $T_{\mathrm{WFM}}=8 \mathrm{~K}$ and above, no such phase transition is evident, as both intensity and wave vector varies continuously as shown in Fig. 5. This underlines, that the magnetoelastic phases at low temperatures correspond to the magnetic phases determined via magnetometry. This is confirmed by detailed studies of the field and temperature dependence of the magnetoelastic response with $\mathrm{x}$ rays in the entire phase diagram. During the course of several x-ray experiments, we monitored both the modulation and peak intensity as a function of field and temperature. The results are summarized in Fig. 6 and are compared with the phase lines depicted in Fig. 2. This comparison shows a strong correlation between changes in both the position, Fig. 6(a), and in the intensity, Fig. 6(b), of the X-ray scattering peak with the phase transitions identified in the SQUID measurements.

\section{DISCUSSION}

$\mathrm{TbNi}_{2} \mathrm{~B}_{2} \mathrm{C}$ like $\mathrm{ErNi}_{2} \mathrm{~B}_{2} \mathrm{C}$ exhibits the so-called "four-state clock"-behavior in the $(a b)$-plane at low temperatures, where the moments are confined to be along either an $a$ or a $b$ axis, with a high-field magnetization along [110] roughly $\sqrt{2}$ smaller than along [100] [35]. Thus, both the magnetic anisotropies and the magnetic ordering vectors are similar in the two systems. The classical dipole-dipole interaction favors the polarization of the ordered moments to be transverse to an ordering vector along $\boldsymbol{a}^{*}$, and this is the polarization observed in $\mathrm{ErNi}_{2} \mathrm{~B}_{2} \mathrm{C}[9,41]$. It is therefore highly surprising that the magnetic polarization is found to be longitudinal in the case of $\mathrm{TbNi}_{2} \mathrm{~B}_{2} \mathrm{C}$ [37]. The classical dipole-dipole interaction relative to the Heisenberg interaction is the factor 1.56/6.25 smaller in $\mathrm{TbNi}_{2} \mathrm{~B}_{2} \mathrm{C}$ than in $\mathrm{ErNi}_{2} \mathrm{~B}_{2} \mathrm{C}$, but still important. Thus, to explain the longitudinal polarization in $\mathrm{TbNi}_{2} \mathrm{~B}_{2} \mathrm{C}$, all the way up to $T_{N}$, an anisotropic spin-spin interaction larger than $10 \%$ of the Heisenberg interaction is needed to overrule the classical dipole-dipole interaction. The magnetoelastic coupling responsible for the orthorhombic distortion does not distinguish between a longitudinal or a transverse polarization of the magnetic moments in the $(a b)$ plane. It is two to

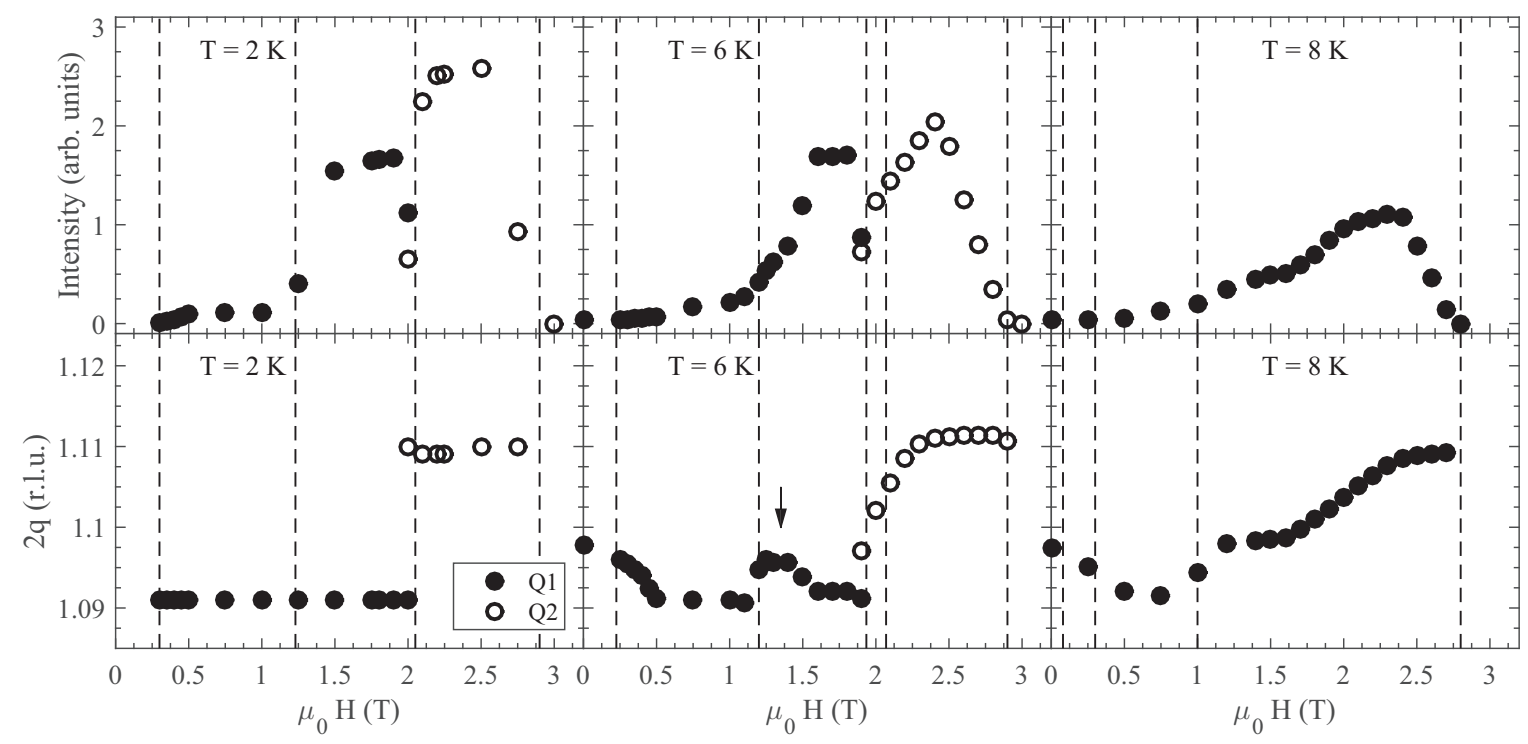

FIG. 5. Field scans for $\boldsymbol{H} \| \boldsymbol{a}$. (Top) Integrated satellite peak intensities at three example temperatures. The vertical lines mark the magnetic phase boundaries determined by the magnetization measurements. The error bars are smaller than the marker sizes. (Bottom) Field dependence of the $2 \boldsymbol{q}$ peak position. 

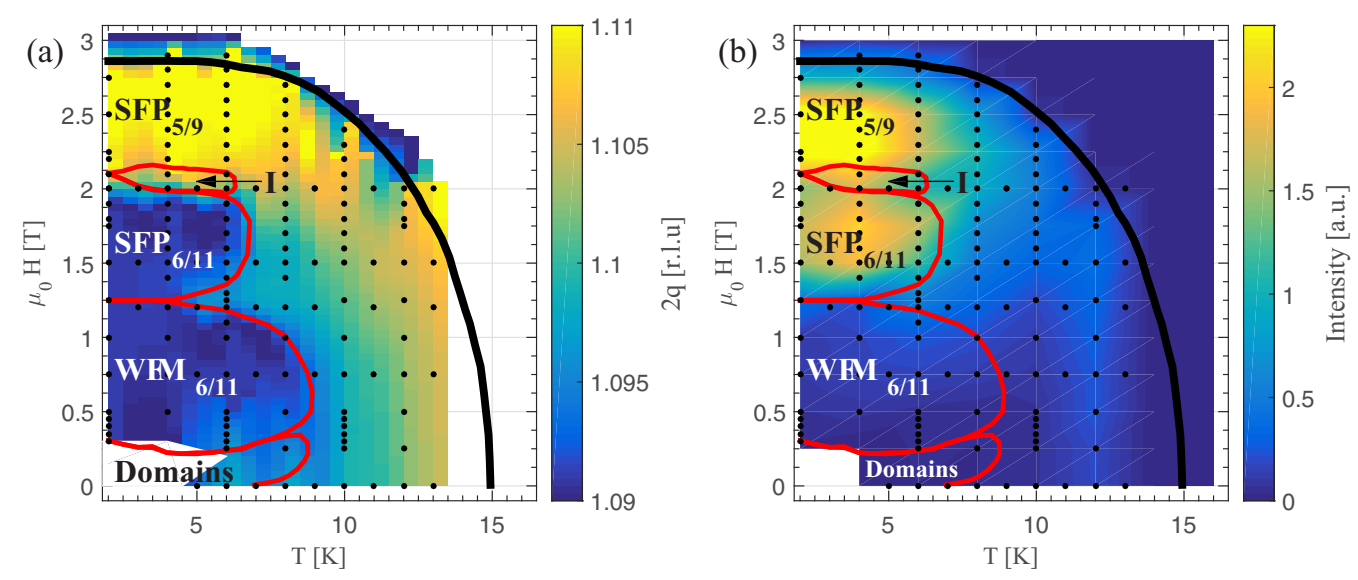

FIG. 6. Phase diagram of $\mathrm{TbNi}_{2} \mathrm{~B}_{2} \mathrm{C}$ as measured via the $\mathrm{x}$-ray peaks probing the magnetoelastic modulation of the crystal at $(2 q, 0,0)$. (a) Variation of the peak position as a function of temperature and applied field-two peaks where observed at the phase boundary near 2 T. (b) Variation of the peak integrated intensity in the $(H, T)$ phase diagram. The solid lines are the phase lines shown in Fig. 2 derived from the magnetization measurements.

three times stronger in $\mathrm{TbNi}_{2} \mathrm{~B}_{2} \mathrm{C}$ than in the Er compound [38], but still relatively small, energetically, compared to the crystal-field anisotropies or the exchange interaction.

The strong confinement at low temperatures of the moments to be along [100] or [010] implies that the magnetic moments are ordered in linearly polarized square waves, which structures favor the ordering wave vector to lock-in to values commensurable with the lattice. In $\mathrm{ErNi}_{2} \mathrm{~B}_{2} \mathrm{C}$ a range of commensurate structures were found throughout the phase diagram with wave vectors $\boldsymbol{q}$ parallel to the $a$ axis [9]. These consist of alternating ferromagnetic $(b c)$-layers with moments pointing either parallel $\uparrow$ or antiparallel $\downarrow$ to the $b$ axis. A simple $q=\frac{1}{2}$ structure is described as $\uparrow \uparrow \downarrow \downarrow \uparrow \uparrow \downarrow \downarrow$ and so on. Structures with larger $q$ are formed by replacing a number of $\uparrow \uparrow(\downarrow \downarrow)$ double layers with a single $\uparrow(\downarrow)$ layer, the so-called spin-slip structures [42].

The Er compound shows a transition to weak ferromagnetism at $T_{\mathrm{WFM}}(\mathrm{Er}) \simeq 2.3 \mathrm{~K}$, which was found to be a transition between two different commensurable spin-slip structures with the same period of 40 layers $[7,41]$. In the case of $\mathrm{TbNi}_{2} \mathrm{~B}_{2} \mathrm{C}$, the zero-field neutron diffraction experiments [37] show that the ordering wave vector $\boldsymbol{q}=(q, 0,0)$ changes linearly with temperature, from $q \simeq 0.552$ at $T_{N}$ until $q$ locks-in to the value 0.545 at $T_{\mathrm{WFM}}$ staying constant below this temperature. Hence, the transition at $T_{\mathrm{WFM}}$ is a transition from a phase with a continuously varying, incommensurable ordering wave vector to a phase with a constant, commensurable $q=\frac{6}{11}$. Two relevant possibilities [43] for the commensurable $q=\frac{6}{11}$ structure, named $q_{2}(\uparrow, \uparrow)$ and $q_{2}(\uparrow, \downarrow)$, are presented in Ref. [37]. Different spin-slip structures with the same period are the more stable the more uniform the spin-slips are distributed. This general principle indicates that $q_{2}(\uparrow, \uparrow)$ should be the stable one of the two structures. This choice agrees with that derived from the analysis of the diffraction results including all higher harmonics, when assuming the structure to be completely squared up [37]. The second, smaller domain, which becomes visible below $10 \mathrm{~K}$ in the diffraction experiment [37], is less well-defined. $q$ changes with temperature all the way down to $1 \mathrm{~K}$, and the scattering peak from the fifth harmonic is smaller and broader than the one deriving from the main domain.
The minority domain might very well be ordered in spin-slip structures, but with an irregular distribution of the spin-slip layers. The irregularities would allow a smooth variation of $q$ and would explain the broadening of the scattering peak. We remark, that it is very unlikely that the two ordering wave vectors, $\boldsymbol{q}=(0.545,0,0)$ and $\boldsymbol{q}=(0.550, \pm 0.002,0)$ at $1 \mathrm{~K}$, derive from a double- $\boldsymbol{q}$ structure in a single domain. There are no similarities between the temperature dependencies of the two wave vectors, and the strong four-state clock anisotropy only allows the moments to jump from being along one $\langle 100\rangle$ direction to the next, nothing like the smooth rotation of the moments required to stabilize a double- $\boldsymbol{q}$ structure [44].

The present experiments at $2 \mathrm{~K}$ show a step-wise behavior of the magnetization with two plateaus below $2 \mathrm{~T}$, where the ordering wave vector is locked to the value $q=0.545$ (see Figs. 1 and 5). The combination of the results for the magnetization and for the field dependence of the ordering wave vector indicates the following sequence of commensurable magnetic structures at $2 \mathrm{~K}$ :

Between 0.3 and $1.3 \mathrm{~T}$ :

$$
\mathrm{WFM}_{6 / 11}=|\downarrow \uparrow \uparrow \downarrow \downarrow \uparrow \uparrow \downarrow \downarrow \uparrow \uparrow\rangle, \quad M \simeq \frac{0.9 \mu_{B}}{\mathrm{~Tb}^{3+}},
$$

Between 1.3 and $2.1 \mathrm{~T}$ :

$$
\mathrm{SFP}_{6 / 11}=|\downarrow \uparrow \uparrow \downarrow \uparrow \uparrow \uparrow \downarrow \downarrow \uparrow \uparrow\rangle, \quad M \simeq \frac{2.6 \mu_{B}}{\mathrm{~Tb}^{3+}},
$$

Between 2.1 and $2.8 \mathrm{~T}($ at $\sim 2.3 \mathrm{~T})$ :

$$
\mathrm{SFP}_{5 / 9}=|\downarrow \uparrow \uparrow \downarrow \uparrow \uparrow \uparrow \downarrow \uparrow \uparrow \uparrow \downarrow \uparrow \uparrow \downarrow \uparrow \uparrow \uparrow\rangle, \quad M \simeq \frac{4.2 \mu_{B}}{\mathrm{~Tb}^{3+}},
$$

where SFP denotes the commensurate spin-flip states above $1.3 \mathrm{~T}$. The magnetization, $M$, is estimated by assuming the structures to be completely squared up with the length of the moments being equal to the saturation value $9.5 \mu_{B}$. These proposed structures imply values for magnetization perfectly in line with those observed and shown in Fig. 1. An additional, small kink in the magnetization curves recorded between 3 and $5 \mathrm{~K}$, indicates an intermediate phase between the $\mathrm{SFP}_{6 / 11}$ and $\mathrm{SFP}_{5 / 9}$ phases named $I$ in Fig. 6, which is not detected in the $\mathrm{x}$-ray scattering experiments. We conjecture this to 
be a phase with the same wave vector, $q=5 / 9$, but with less spins flipped compared to the $\mathrm{SFP}_{5 / 9}$ phase. The path to full magnetization for the 18-layer structure in the spin-flip phase $\mathrm{SFP}_{5 / 9}$ would go through a successive reduction of the number of antiparallel moments. The $\mathrm{WFM}_{6 / 11}$ structure is the same as the weakly ferromagnetic $q_{2}(\uparrow, \uparrow)$ structure derived from the neutron diffraction experiments at zero field [37]. The magnetization data at $2 \mathrm{~K}$ shown in Fig. 1 are obtained after cooling in zero field implying that the crystal is divided into twinning domains, $\boldsymbol{q}$ may be along $\boldsymbol{b}^{*}$ as well as along $\boldsymbol{a}^{*}$, before the field is applied, which domains are being stabilized by the corresponding orthorhombic distortions. When applying the field along $\boldsymbol{a}$, the SQUID measurements indicate a gradual disappearance of the unfavorable domains polarized along $\boldsymbol{b}$ and a transition to a single $\boldsymbol{a}$ domain at about $\mu_{0} H=0.3 \mathrm{~T}$, with a resulting plateau in magnetization. At higher temperatures, above $T_{\mathrm{WFM}}=8 \mathrm{~K}$, the modulation of the magnetic structures becomes incommensurable, and as shown in Fig. 6(a), $2 q$ changes in a continuous way with both field and temperature. This is accompanied by a smooth variation of the magnetization as shown by the example at $T=10 \mathrm{~K}$ in Fig. 1.

The orthorhombic distortion of $\mathrm{TbNi}_{2} \mathrm{~B}_{2} \mathrm{C}$ below $T_{N}$ is described by the following Hamiltonian $[19,41,45]$ for the $i$ th Tb ion:

$$
\mathcal{H}_{i}^{\mathrm{me}}=-B_{\gamma}\left(\epsilon_{11}-\epsilon_{22}\right)_{i} O_{2}^{2}(i)+\frac{c_{11}-c_{12}}{4 N}\left(\epsilon_{11}-\epsilon_{22}\right)_{i}^{2}
$$

where $O_{2}^{2}(i)=\left(J_{x}^{2}-J_{y}^{2}\right)_{i}$ is the Stevens operator at the site $i$ with $\hat{\boldsymbol{x}}$ and $\hat{\boldsymbol{y}}$ being along, respectively, the $a$ and $b$ axis. The magnetoelastic effects due to the uniform strain component $\left\langle\left(\epsilon_{11}-\epsilon_{22}\right)_{i}\right\rangle=1-b / a$ has been studied by El Massalami et al. [19] Besides giving rise to the observed orthorhombic deformation of the crystal in the ordered phase below $T_{N}$, this Hamiltonian produces a longitudinal deformation $E_{11}\left(\boldsymbol{R}_{i}\right)$, corresponding to a modulation of $\epsilon_{11}$ when $\boldsymbol{q} \| \boldsymbol{a}^{*}$, where the reduced elastic constant $\left(c_{11}-c_{12}\right) / 2 N$ should be replaced by the one deriving from the corresponding elastic deformation. If $\left\langle J_{x}(i)\right\rangle$ is described by a single wave vector $\boldsymbol{q}$ (no higher harmonics) the modulated deformations, being proportional to the squared moments, only occur at the wave vector $2 \boldsymbol{q}$. In the case where the higher harmonics become important, other higher harmonics are also going to appear in the elastic modulation of the crystal, but the second harmonic is likely always going to be the dominating one, as is the case in the present system. However, it is important to realize, that in the limit of complete squaring up, the squared moments, or $\left\langle O_{2}^{2}(i)\right\rangle$, become independent of the site considered and the modulated deformations vanish leaving only the uniform one.

Figure 6(b) shows the intensity of the x-ray diffraction peak throughout the phase diagram, which intensity reflects the square of the amplitude of the deformations. The high intensity region is confined to the spin-flip phases $\mathrm{SFP}_{6 / 11}$ and $\mathrm{SFP}_{5 / 9}$ at low temperatures and high fields. The amplitude of the deformation at $2 \boldsymbol{q}$ is determined by the second harmonic of $\left\langle O_{2}^{2}(i)\right\rangle$ and the energy of the longitudinal phonon at this wave vector. It seems unlikely that the optical, longitudinal phonon close to $(1,0,0)$ should become soft when applying a field. The softening observed in the borocarbides is occurring around $(0.5,0,0)$ and involves dominantly the transverse polarization of the phonons [40]. Hence, the large jump in the scattering intensity at $1.3 \mathrm{~T}$ must reflect a large jump in the second harmonic of $\left\langle O_{2}^{2}(i)\right\rangle$. This second harmonic vanishes in the case of the simple $\left(\frac{1}{2}, 0,0\right)$ structure, or if the magnetic structure is completely squared up. The $\mathrm{WFM}_{6 / 11}$ structure contains a single spin-slip layer per 11 layers and is close to fulfill both criteria at $2 \mathrm{~K}$. The introducing of additional spin flips is going to increase the second harmonic, because of the larger variation in the size of the magnetic moments close to spin-slip layers or layers where the spins are about to flip. Hence, an increase of the second harmonic is expected to occur at the transition from $\mathrm{WFM}_{6 / 11}$ to $\mathrm{SFP}_{6 / 11}$ and once again at the next transition from $\mathrm{SFP}_{6 / 11}$ to $\mathrm{SFP}_{5 / 9}$. As shown in Fig. 4, the increase in the scattering intensity at the last transition looks reasonable, whereas the increase occurring at the first transition is an order of magnitude larger than derived from any reasonable estimate.

We have done some calculations on a model, which should give a reasonable description of $\mathrm{TbNi}_{2} \mathrm{~B}_{2} \mathrm{C}$. The model corresponds to the one applied in the case of $\mathrm{ErNi}_{2} \mathrm{~B}_{2} \mathrm{C}$ [41], except that the classical dipole-dipole interaction has been replaced by an effective anisotropic dipole-dipole interaction 10 times smaller but with the opposite sign of the classical one. This means that the cases of transverse and longitudinal polarization of the ordered moments are nearly degenerate with a slight favoring of the longitudinal polarization. Because of the small difference between the two cases, the calculations suggest another possibility for, for instance, the structure in the intermediate phase at $2 \mathrm{~K}$ :

Between 1.3 and $2.1 \mathrm{~T}$ :

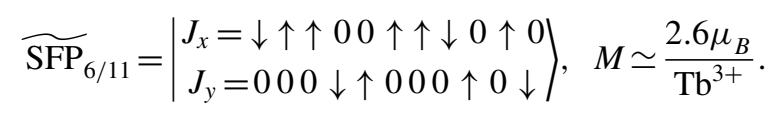

This is a noncollinear structure, where the $a$-axis moments in successive layers along the $a$ axis are either $\uparrow, \downarrow$, or 0 . Whenever the $a$ component is 0 the $b$ component is either $\uparrow$ or $\downarrow$, elsewhere the $b$ component is 0 . Incidentally, the calculated moments are not fully polarized nor exactly confined to be along an $a$ or $b$ axis, but these modifications are not important for the present arguments. The magnetization is the same in the two structures, whereas the quadrupolar second harmonic is a factor 10 larger in the $\widetilde{\mathrm{SFP}}_{6 / 11}$ phase compared to that derived for the collinear $\mathrm{SFP}_{6 / 11}$ structure. Hence, the drastic change in the $\mathrm{x}$-ray peak intensity at the transition at $1.3 \mathrm{~T}$ might be explained by assuming that the moments above $\mu_{0} H=1.3 \mathrm{~T}$ are ordered in noncollinear ways, like the one presented in Eq. (4). However, the magnetostriction measurements made by El Massalami et al. [19] disqualify this explanation. A strong enhancement of the second harmonic is necessarily going to be accompanied by a reduction of the uniform component of $\left\langle\mathrm{O}_{2}^{2}(i)\right\rangle$, by a factor estimated to be about 3 in the present case. The forced magnetostriction measurements made at 3-5 K show only small kinks, no large jumps, at the transitions between the different phases [19]. These results indicate that the x-ray peak intensities observed above $1.3 \mathrm{~T}$ are in accordance with the collinear structures proposed in Eq. (1), but that it is the intensity observed below $1.3 \mathrm{~T}$ which is much smaller than should be expected. The maximum in $\mathrm{x}$-ray scattering intensity at $2 \mathrm{~K}$ is found to correspond to a modulated strain $E_{11}(2 q)$ of about $7 \cdot 10^{-4}$, nearly a factor of 
10 smaller than the homogeneous strain $1-b / a$ observed [37] at this temperature. The ratio of 10 between the two strains is close to the calculated ratio between the uniform component and the second harmonic of the quadrupolar moment in the $\mathrm{SFP}_{5 / 9}$ phase derived by the model calculations. The large intensities observed above $1.3 \mathrm{~T}$ is actually quite reasonable, when being compared to the large orthorhombic deformation of the system. Hence, the absolute magnitudes of the intensities, once more, indicates that it is the scattering intensity below $1.3 \mathrm{~T}$ which is unexpectedly small. The reason for this reduced intensity is, either (i) the modulation of the ordered moments at $2 \mathrm{~K}$ is much closer to a square wave than indicated by the model calculations, or (ii) that the second-harmonic part of the $\mathrm{x}$-ray scattering intensity appears somewhere else at low fields. There are indications that the crystal is divided in domains with different ordering wave vectors and that $\boldsymbol{q}$ is rotated slightly away from the $a$ direction [37].

\section{CONCLUSION}

In conclusion, we perform a thorough examination of the magnetic and magnetoelastic phase diagram of $\mathrm{TbNi}_{2} \mathrm{~B}_{2} \mathrm{C}$. Extensive SQUID measurements outline the magnetic phase boundaries of this complex phase diagram to a previously unmatched degree of detail. Synchrotron hard x-ray measurements were used to measure the magnetoelastic deformations throughout the phase diagram. The wave vectors of these deformations strongly suggest that the magnetic structures giving rise to them are commensurate spin-slip-type structure similar to those found in $\mathrm{ErNi}_{2} \mathrm{~B}_{2} \mathrm{C}$. Suggestions for these magnetic structures were made that give rise to both the correct wave vectors of the elastic deformations and to the observed magnetization. The rough field dependence of the amplitudes of the deformations can be explained by the second harmonic of the quadrupole moment, but the reason for the violent jump in the $\mathrm{x}$-ray scattering intensity at $1.3 \mathrm{~T}$ remains to be investigated and is subject to further studies.

\section{ACKNOWLEDGMENTS}

This work was supported by the Danish Agency for Science, Technology, and Innovation under DANSCATT. X-ray experiments were performed at DESY. SQUID measurements were perform the at the Helmholtz-Zentrum Berlin für Materialien und Energie (HZB). We thank HZB for access to the magnetization equipments and DESY for the allocation of synchrotron radiation beam time.
[1] R. J. Cava, H. Takagi, H. W. Zandbergen, J. J. Krajewski, W. F. Peck, Jr., T. Siegrist, B. Batlogg, R. B. van Dover, R. J. Felder, K. Mizuhashi, J. O. Lee, H. Eisaki, and S. Uchida, Nature 367, 252 (1994).

[2] R. J. Cava, H. W. Zandbergen, B. Batlogg, H. Eisaki, H. Takagi, J. J. Krajewski, W. F. Peck, Jr., E. M. Gyorgy, and S. Uchida, Nature 372, 245 (1994).

[3] R. Nagarajan, C. Mazumdar, Z. Hossain, S. K. Dhar, K. V. Gopalakrishnan, L. C. Gupta, C. Godart, B. D.Padalia, and R. Vijayaraghavan, Phys. Rev. Lett. 72, 274 (1994).

[4] T. Siegrist, H. W. Zandbergen, R. J. Cava, J. J. Krajewski, and W. F. Peck, Jr., Nature 367, 254 (1994).

[5] H. Eisaki, H. Takagi, R. J. Cava, B. Batlogg, J. J. Krajewski, W. F. Peck, Jr., K. Mizuhashi, J. O. Lee, and S. Uchida, Phys. Rev. B 50, 647 (1994).

[6] J. Jensen and P. Hedegård, Phys. Rev. B 76, 094504 (2007).

[7] S.-M. Choi, J. W. Lynn, D. Lopez, P. L. Gammel, P. C. Canfield, and S. L. Bud'ko, Phys. Rev. Lett. 87, 107001 (2001).

[8] H. Kawano-Furukawa, H. Takeshita, M. Ochiai, T. Nagata, H. Yoshizawa, N. Furukawa, H. Takeya, and K. Kadowaki, Phys. Rev. B 65, 180508 (2002).

[9] A. Jensen, K. Nørgaard Toft, A. B. Abrahamsen, D. F. McMorrow, M. R. Eskildsen, N. H. Andersen, J. Jensen, P. Hedegård, J. Klenke, S. Danilkin, K. Prokes, V. Sikolenko, P. Smeibidl, S. L. Bud'ko, and P. C. Canfield, Phys. Rev. B 69, 104527 (2004).

[10] A. Jensen, K. Nørgaard Toft, A. B. Abrahamsen, N. H. Andersen, J. Jensen, P. Hedegård, J. Klenke, K. Prokes, P. Smeibidl, S. Danilkin, V. Sikolenko, M. R. Eskildsen, and P. C. Canfield, Physica C 408-410, 97 (2004).

[11] P. C. Canfield, S. L. Bud'ko, and B. K. Cho, Physica C 262, 249 (1996).
[12] U. Yaron, P. L. Gammel, A. P. Ramirez, D. A. Huse, D. J. Bishop, A. I. Goldman, C. Stassis, P. C. Canfield, K. Mortensen, and M. R. Eskildsen, Nature 382, 236 (1996).

[13] V. L. Ginzburg, Sov. Phys. JETP 4, 153 (1957).

[14] J. W. Lynn, G. Shirane, W. Thomlinson, and R. N. Shelton, Phys. Rev. Lett. 46, 368 (1981).

[15] S. S. Saxena, P. Agarwal, K. Ahilan, F. M. Grosche, R. K. W. Haselwimmer, M. J. Steiner, E. Pugh, I. R. Walker, S. R. Julian, P. Monthoux, G. G. Lonzarich, A. Huxley, I. Sheikin, D. Braithwaite, and J. Flouquet, Nature 406, 587 (2000).

[16] J. Wang, M. Singh, M. Tian, N. Kumar, B. Liu, C. Shi, J. K. Jain, N. Samarth, T. E. Mallouk, and M. H. W. Chan, Nat. Phys. 6, 389 (2010).

[17] M. El Massalami, H. Takeya, K. Hirata, M. Amara, R.-M. Galera, and D. Schmitt, Phys. Rev. B 67, 144421 (2003).

[18] M. Doerr, M. Rotter, M. El Massalami, S. Sinning, H. Takeya, and M. Loewenhaupt, J. Phys.: Condens. Matter 14, 5609 (2002).

[19] M. El Massalami, M. Amara, R.-M. Galéra, D. Schmitt, and H. Takeya, Phys. Rev. B 76, 104410 (2007).

[20] P. Dervenagas, J. Zarestky, C. Stassis, A. I. Goldman, P. C. Canfield, and B. K. Cho, Phys. Rev. B 53, 8506 (1996).

[21] J. W. Lynn, S. Skanthakumar, Q. Huang, S. K. Sinha, Z. Hossain, L. C. Gupta, R. Nagarajan, and C. Godart, Phys. Rev. B 55, 6584 (1997).

[22] J. Y. Rhee, X. Wang, and B. N. Harmon, Phys. Rev. B 51, 15585 (1995).

[23] S. B. Dugdale, M. A. Alam, I. Wilkinson, R. J. Hughes, I. R. Fisher, P. C. Canfield, T. Jarlborg, and G. Santi, Phys. Rev. Lett. 83, 4824 (1999).

[24] N. H. Andersen, J. Jensen, T. B. S. Jensen, M. V. Zimmermann, R. Pinholt, A. B. Abrahamsen, K. N. Toft, P. Hedegård, and P. C. Canfield, Phys. Rev. B 73, 020504(R) (2006). 
[25] K. Nørgaard Toft, A. B. Abrahamsen, M. R. Eskildsen, K. Lefmann, N. H. Andersen, P. Vorderwisch, P. Smeibidl, M. Meissner, and P. C. Canfield, Phys. Rev. B 69, 214507 (2004).

[26] P. Dervenagas, M. Bullock, J. Zarestky, P. Canfield, B. K. Cho, B. Harmon, A. I. Goldman, and C. Stassi, Phys. Rev. B 52, R9839(R) (1995).

[27] H. Kawano, H. Yoshizawa, H. Takeya, and K. Kadowaki, Phys. Rev. Lett. 77, 4628 (1996).

[28] M. Bullock, J. Zarestky, C. Stassis, A. Goldman, P. Canfield, Z. Honda, G. Shirane, and S. M. Shapiro, Phys. Rev. B 57, 7916 (1998).

[29] C. V. Tomy, L. A. Afalfiz, M. R. Lees, J. M. Martin, D. M. Paul, and D. T. Adroja, Phys. Rev. B 53, 307 (1996).

[30] H. Bitterlich, W. Loser, G. Behr, K. Nenkov, G. Fuchs, A. Belger, and L. Schultz, Physica C 308, 243 (1998).

[31] U. Jaenicke-Roessler, A. Belger, G. Zahn, B. Wehner, P. Paufler, and H. Bitterlich, Physica C 314, 43 (1999).

[32] C. V. Tomy, L. J. Chang, D. Paul, and C. Ritter, Physica B 230232, 872 (1997).

[33] C. Song, D. Wermeille, A. I. Goldman, P. C. Canfield, J. Y. Rhee, and B. N. Harmon, Phys. Rev. B 63, 104507 (2001).

[34] M. B. Fontes, J. C. Trochez, B. Giordanengo, S. L. Bud'ko, D. R. Sánchez, E. M. Baggio-Saitovitch, and M. A. Continentino, Phys. Rev. B 60, 6781 (1999).
[35] B. K. Cho, P. C. Canfield, and D. C. Johnston, Phys. Rev. B 53, 8499 (1996).

[36] D. R. Sánchez, M. A. C. de Melo, M. B. Fontes, S. L. Bud'ko, E. Baggio-Saitovitch, M. Hillberg, W. Wagener, H. H. Klauß, G. H. Walf, and F. J. Litterst, Phys. Rev. B 57, 10268 (1998).

[37] H. Kawano-Furukawa, H. Tsukagoshi, T. Nagata, C. Kobayashi, H. Yoshizawa, and H. Takeya, Phys. Rev. B 77, 144426 (2008).

[38] C. Song, Z. Islam, L. Lottermoser, A. I. Goldman, P. C. Canfield, and C. Detlefs, Phys. Rev. B 60, 6223 (1999).

[39] A. Kreyssig, O. Stockert, D. Reznik, F. M. Woodward, J. W. Lynn, W. Reichardt, D. Souptel, G. Behr, and M. Loewenhaupt, Physica B 350, 69 (2004).

[40] S. Anissimova, A. Kreyssig, O. Stockert, M. Loewenhaupt, and D. Reznik, Phys. Rev. B 84, 104509 (2011).

[41] J. Jensen, Phys. Rev. B 65, 140514(R) (2002).

[42] D. Gibbs, D. E. Moncton, K. L. D'Amico, J. Bohr, and B. H. Grier, Phys. Rev. Lett. 55, 234 (1985).

[43] The "nodal structures" with nonmagnetic layers presented in Ref. [37], as for instance the one named $q_{2}(0,0)$, are completely unstable.

[44] J. Jensen and M. Rotter, Phys. Rev. B 77, 134408 (2008).

[45] J. Jensen and A. R. Mackintosh, Rare Earth Magnetism: Structures and Excitations (Clarendon, Oxford, 1991), http://www.nbi.ku.dk/page40667.htm. 